\title{
Josephson Effect in Coherent Roton Aggregates
}

\author{
Lev A. Melnikovsky* \\ P.L. Kapitza Institute for Physical Problems \\ Russian Academy of Sciences, Moscow, Russia
}

\begin{abstract}
A microwave electromagnetic field can excite a coherent roton aggregate in liquid helium around a dielectric resonator. We show that multiple coherent aggregates are excited simultaneously and predict a Josephson effect between them. The superfluid velocity acts as a "voltage across the weak link" in superconducting Josephson junctions. A comparison with existing experimental data is made.
\end{abstract}

It was shown earlier [1, 2, that a Coherent Aggregate of Roton Pairs (CARP) in liquid helium- 4 around a dielectric resonator can be excited by an electromagnetic field in the microwave range. Experimentally, the CARP build-up manifests [3] as an ultra-narrow peak in the resonator loss at the frequency of $f_{0}=\Delta /(2 \pi \hbar) \sim 180 \mathrm{GHz}$, where $\Delta$ is the roton energy gap. Coupling of the microwave radiation to the CARP is due to the dependence of individual roton energy $\varepsilon$ on the electric field $\mathbf{E}(t)$ :

$$
\delta \varepsilon \sim \alpha \frac{E^{2}}{2},
$$

where $\alpha$ is the roton polarizability. Elementary process of such parametric excitation is the transformation of two photons into two rotons. Energy of the photon pair $4 \pi \hbar f$ in the initial state is equal to the energy of the roton pair $2 \varepsilon=2 \Delta$. The photon momentum was earlier [2] neglected because it is much smaller than that of a roton. This momentum is in fact responsible for an interesting effect described below.

Electromagnetic field in a whispering gallery disk resonator is a superposition of two traveling modes - a counterclockwise wave and a clockwise wave. The azimuthal projection of the wave vector for these modes is

$$
k= \pm \frac{N}{R}
$$

where $R$ is the resonator radius and $N$ is the mode number. The azimuthal momentum of the roton pair may therefore take one of three possible values

$$
p_{-}=-\frac{2 \hbar N}{R}, \quad p_{0}=0, \quad \text { or } \quad p_{+}=\frac{2 \hbar N}{R} .
$$

*E-mail: leva@kapitza.ras.ru 
This means that actually three CARPs are excited by the parametric resonance rather than just one. Suppose a weak interaction exists between these coherent particle reservoirs. The internal Josephson effect is to be expected in such system. The Josephson currents between the CARPs would be determined by the phase differences between them.

Roton pairs of different azimuthal momenta in a motionless liquid have equal energy $2 \Delta$. This degeneracy may be removed by a superfluid velocity $v_{\mathrm{s}}$. Imagine an axially symmetric vortex superflow tangential to the disk circumference. The energy of the roton pair then becomes

$$
2 \varepsilon_{\{-, 0,+\}}=2 \Delta+v_{\mathrm{s}} p_{\{-, 0,+\}} .
$$

Note, that $v_{\mathrm{s}}$ here is not arbitrary, it depends on the number of quantized vortices $n$ pinned by the resonator:

$$
v_{\mathrm{s}}=\frac{n \hbar}{R m_{\mathrm{He}}} .
$$

The time-dependant parts of the phase differences are simply $\Omega_{n} t$ and $2 \Omega_{n} t$, where

$$
\Omega_{n}=\frac{v_{\mathrm{s}} p_{+}}{\hbar}=\frac{2 \hbar N n}{R^{2} m_{\mathrm{He}}}=2 \pi n \cdot 0.0043 \mathrm{~Hz} .
$$

Here $R=9.5 \mathrm{~mm}$ and $N=78$ (see [4]). With the frequency $\Omega_{n}$ both the Josephson currents and CARPs population oscillate. The latter is directly probed in microwave experiments. Indeed, the step-like behavior and the low frequency modulation of the resonator loss have been observed [4. In this experiment the superfluid circulation around the disk was generated by two "heat guns" and the modulation amplitude changed stepwise with continuous increase of the gun power. These steps can be attributed to the circulation quantization. It should be possible to extract the oscillation frequency step corresponding to one vortex quantum. Unfortunately, available data 4 leave certain amount of uncertainty about exact value of this step, it is probably confined within the range $0.002 \mathrm{~Hz}-0.04 \mathrm{~Hz}$ in good agreement with (1).

It is possible that small Josephson oscillation are masked in experiment by some spurious beat-frequency interference between electromagnetic waves absorbed by CARPs of different momenta. The lowest beat frequency is $\Omega_{n} / 2$.

I thank A.F. Andreev, V.I. Marchenko, A.S. Rybalko, and A.I. Smirnov for fruitful discussions. This work was supported in parts by RF president program NSh-4889.2012.2 and RFBR grants 13-02-00912 and 13-02-90494.

\section{References}

[1] L.A. Melnikovsky, JETP Lett. 96, 98 (2012).

[2] L.A. Melnikovsky, arXiv:1206.4592, JLTP 171, 234 (2013).

[3] A. Rybalko, S. Rubets, E. Rudavskii, V. Tikhiy, S. Tarapov, R. Golovashchenko, and V. Derkach, Phys. Rev. B76 140503 (2007).

[4] A.S. Rybalko, S.P. Rubets, E.Ya. Rudavskii, V.A. Tikhii, S.I. Tarapov, R.V. Golovashchenko, and V.N. Derkach, Low Temp. Phys. 34, 497 (2008). 